\title{
Estructura de Plan de Continuidad Operativa Bajo el Enfoque de la Gestión de Riesgo de Desastres en Empresas de Saneamiento de Agua
}

\section{STRUCTURE OF BUSINESS CONTINUITY PLAN UNDER THE APPROACH OF DISASTER RISK MANAGEMENT IN PUBLIC WATER COMPANIES}

Pablo César Gutiérrez Falcón'

1. Universidad Nacional Mayor de San Marcos. Universidad Tecnológica del Perú, Lima - Perú.

\section{RESUMEN}

En el marco del Sistema Nacional de Gestión de Riesgos de Desastres, el Estado Peruano ha establecido la necesidad de desarrollar la Gestión de la Continuidad Operativa en todos los niveles de gobierno. A partir de ello, nace la obligación que las entidades públicas deban elaborar Planes de Continuidad Operativa; sin embargo, no se cuenta con referencias documentarias ni técnicas que permitan servir de información de entrada para dichos planes. Actualmente, existe abundante normativa técnicas e investigaciones que desarrollan la continuidad operativa (continuidad de negocio) bajo el enfoque de las tecnologías de la información.

La presente investigación consistió en la revisión de documentación normativa, técnica y de benchmark (modelos empresariales) con el propósito de diseñar la estructura modelo que debería comprender un Plan de Continuidad Operativa.

Dicha estructura modelo fue implementada en una empresa de servicios de saneamiento, permitiéndole abordar las amenazas de riesgo operativo en los macroprocesos y procesos de Nivel 1 considerados como prioritarios.

(Gutiérrez P, 2018. Estructura de Plan de Continuidad Operativa Bajo el Enfoque de la Gestión de Riesgo de Desastres en Empresas de Saneamiento de Agua. Cienc Trab. Sep-Dic; 20 [63]: 169-177).

Palabras clave: CONTINUIDAD OPERATIVA, CONTINUIDAD DEL NEGOCIO, GESTIÓN DE RIESGOS, DESASTRE INDUSTRIAL.

\section{ABSTRACT}

According to the National Disaster Risk Management System, the Peruvian State has established the need to develop the Business Continuity Management at all levels of government. From this, the obligation that the public entities must elaborate Business Continuity Plans is born; however, there are no documentary references or techniques that can serve as input information for these plans. Currently, there are abundant technical regulations and investigations that develop business continuity under the focus of information technologies.

The present investigation consisted in the revision of normative, technical and benchmark documentation with the purpose of designing the model structure that should comprise an Operational Continuity Plan.

This model structure was implemented in a public water company, allowing it to address the operational risk threats in the macroprocesses and processes Level 1.

Key Words: BUSINESS CONTINUITY, RISK MANAGEMENT, INDUSTRIAL DISASTER.

\section{INTRODUCCIÓN}

En el marco de la Política Nacional de Gestión de Riesgos de Desastres $^{i}$, instrumento de obligatorio cumplimiento por las Entidades Públicas en todos los niveles de gobierno, se busca contribuir con el

Correspondencia / Correspondence:

Sr. Pablo César Gutiérrez Falcón

Av. Belisario Suarez 948 San Juan de Miraflores, Lima - Perú Código Postal: 15801

Tel.: (51-1) 997369253

e-mail 1: pcgutierrezf@gmail.com

e-mail 2: pgutierrezf@copan-sgi.com

Recibido: 23 de septiembre de 2018 / Aceptado: 01 de noviembre de 2018
Sistema Nacional de Gestión de Riesgo de Desastres (SINAGERD); así como con la implementación de la Gestión de la Continuidad Operativa en los tres niveles de gobierno.

En virtud de ello, se aprobó, mediante Resolución Ministerial $N^{\circ}$ 028-2015-PCM, el documento "Lineamientos para la Gestión de Continuidad Operativa de la Entidades Públicas en los Tres Niveles de Gobierno", que establece lineamientos para que las entidades públicas implementen la Gestión de Continuidad Operativa adecuándola a su alcance y a la complejidad de sus operaciones y servicios. Esta gestión se implementa mediante la ejecución del Plan de Continuidad Operativa. Además, establece 4 componentes para la Gestión de la Continuidad Operativa.

${ }^{i}$ Conjunto de orientaciones dirigidas a impedir o reducir los riesgos de desastres, evitar la generación de nuevos riesgos y efectuar una adecuada preparación, atención, rehabilitación y reconstrucción ante situaciones de desastres, así como a minimizar sus efectos adversos sobre la población, la economía y el ambiente (Ley N²9664, artículo 5\%). 
Tabla 1.

Componentes de la Gestión de la Continuidad Operativa según la Resolución Ministerial Nº28-2015-PCM (Perú).

\begin{tabular}{l|l}
\hline Componentes & Elementos \\
$\begin{array}{l}\text { Análisis de riesgo, } \\
\text { y de recursos }\end{array}$ & $\begin{array}{l}\text { Análisis de la misión (propósito) de la organización. } \\
\text { Identificación de procesos, servicios y proveedores. } \\
\text { Evaluación de riesgos } \\
\text { Análisis del impacto } \\
\text { Determinación de las actividades críticas } \\
\text { Determinación de los recursos humanos } \\
\text { Determinación de los recursos informáticos e } \\
\text { información críticos } \\
\text { Determinación de los recursos fisicos criticos }\end{array}$ \\
\hline $\begin{array}{l}\text { Desarrollo e implementación } \\
\text { de la gestión de la } \\
\text { continuidad operativa }\end{array}$ & $\begin{array}{l}\text { Gestión de crisis } \\
\text { Plan de continuidad operativa }\end{array}$ \\
\hline $\begin{array}{l}\text { Pruebas y actualización de los } \\
\text { Planes de Continuidad } \\
\text { Operativa }\end{array}$ & $\begin{array}{l}\text { Ejecución de ensayos y pruebas } \\
\text { Actualización permanente de los planes }\end{array}$ \\
\hline $\begin{array}{l}\text { Integración de la gestión de la } \\
\text { continuidad operativa a } \\
\text { la cultura organizacional }\end{array}$ & $\begin{array}{l}\text { Evaluación del grado de conocimiento sobre la } \\
\text { gestión de la continuidad }\end{array}$ \\
\hline $\begin{array}{l}\text { Fuente: Elaboración Propia. } \\
\text { Desarrollo y mejora de la cultura de continuidad }\end{array}$
\end{tabular}

\section{Aspectos Generales}

La norma ISO 22301-2012 Seguridad de la Sociedad: Sistemas de Continuidad del Negocio - Requisitos define la "Continuidad de Negocio" como la capacidad de la organización de continuar entregando productos y servicios a niveles aceptables predefinidos después de ocurrir un evento alterador.

Por su lado, el Estado Peruano define, en el documento Lineamientos para la Gestión de Continuidad Operativa de la Entidades Públicas en los Tres Niveles de Gobierno, aprobado mediante Resolución Ministerial $\mathrm{N}^{\circ}$ 028-2015-PCM, el "Gestión de Continuidad Operativa del Estado" como el proceso continuo que debe formar parte de las operaciones habituales de la Entidad Pública y tiene como objetivo garantizar que siga cumpliendo con su misión, mediante la implementación de mecanismos adecuados, con el fin de salvaguardar los intereses de la Nación, ante la ocurrencia de un desastre de gran magnitud o cualquier evento que interrumpa o produzca inestabilidad en sus operaciones.

Belaunde $^{1}$ analiza la aplicación de los términos "continuidad de negocio" y "continuidad operativa”. Belaunde señala que, en general, se utiliza el término "continuidad del negocio" en el mundo empresarial; pero en el caso de las entidades estatales (cuyo objeto no es hacer negocios), se usan los términos "continuidad de las operaciones" (o "continuidad operativa"), y "continuidad del Gobierno" (o del Estado). Aunque en algunos organismos se sigue usando la expresión "business continuity" (es decir, continuidad del negocio) para entidades estatales.

La organización Atalait ${ }^{2}$ señala que toda empresa debería saber cómo resolver cualquier tipo de crisis o de interrupción de los procesos que resultan críticos para una organización. Se estima que un 60\% de la inversión realizada en un plan de continuidad operativa tendrá un impacto directo sobre los activos humanos de la empresa. Los recursos humanos son quienes se encargarán de que el ROI llegue de la mejor manera, en tiempo y forma. Para lograrlo es necesario contar con un plan sólido y probado que brinde un retorno de inversión, y asegure la eficiencia financiera.

\footnotetext{
ii El proceso de rehabilitación contempla asegurar la continuidad de los servicios básicos indispensables.
}

Diferencias entre gestión de riesgos de desastres y gestión de la continuidad operativa

La Ley N²9664 - Ley del Sistema Nacional de Gestión de Riesgo de Desastre define la "Gestión del Riesgo de Desastres" como un proceso social cuyo fin último es la prevención, la reducción y el control permanente de los factores de riesgo de desastre en la sociedad, así como la adecuada preparación y respuesta ante situaciones de desastre, considerando las políticas nacionales con especial énfasis en aquellas relativas a materia económica, ambiental, de seguridad, defensa nacional y territorial de manera sostenible. Además, contempla los siguientes procesos:

- Estimación del riesgo,

- Prevención y Reducción del Riesgo,

- Preparación, Respuesta y Rehabilitación ${ }^{i i}, \mathrm{y}$

- Reconstrucción.

El Reglamento de la Ley N²9664 - Ley del Sistema Nacional de Gestión de Riesgo de Desastre, aprobado mediante Decreto Supremo $\mathrm{N}^{\circ}$ 048-2011-PCM, determina la necesidad de desarrollar planes específicos por los procesos mencionados anteriormente, tales como:

- Planes de prevención y reducción de riesgo de desastres,

- Planes de preparación,

- Planes de operaciones de emergencia,

- Planes de educación comunitaria,

- Planes de rehabilitación, y

- Planes de contingencia.

Belaunde $^{1}$ propone que hay un tema de intersección entre la Continuidad Operativa y la Gestión de Riesgos de Desastres, que es los "eventos de desastres". En el Perú, lo que más puede provocar eventos de interrupción serios, son los desastres naturales; sin embargo, la propia historia peruana permite ampliar el alcance del término desastres debido a que se han tenido también épocas de disturbios violentos y de terrorismo. Así también, los procesos de gestión del riesgo de desastres pueden ser imposibles de llevar a cabo si no se tiene una buena gestión de la continuidad operativa, es decir una buena gestión de riesgo de desastre es imposible sin una buena gestión de la continuidad operativa.

También se debe tener en cuenta que, según lo indicado por el Dr. Belaunde, en el marco de la gestión de la continuidad operativa, se desarrollan planes de continuidad, y estos tienen que articularse con los planes de operaciones de emergencia que tengan la organización, porque, entre otros aspectos, puede haber personal involucrado en ambos temas al mismo tiempo.

Es importante para el desarrollo de la presente investigación adoptar el término de Continuidad Operativa, cuando nos referimos a continuidad del negocio, esto alineándose con el marco normativo peruano correspondiente.

\section{Diferencias entre Plan de Operaciones de Emergencia, Plan de Continuidad Operativa y Plan de Contingencia} Es necesario establecer las diferencias entre los siguientes términos que permitirán aclarar y acotar la investigación, debido a que usualmente estos términos se usan en forma indistinta pero que en el ámbito de la continuidad operativa y de la gestión de riesgos de desastres, tienen acepciones distintas:

- Plan de Operaciones de Emergencia,

- Plan de Continuidad Operativa, y

- Plan de Contingencia. 
El Plan de Operaciones de Emergencia, conocido comúnmente como Plan de Emergencia, es definido por el Servicio Nacional de Aprendizaje - SENA, $2013^{3}$ como el conjunto de normas y procedimientos generales destinados a prevenir y a controlar en forma oportuna y adecuada, las situaciones de riesgo de una empresa, y que se basa en el análisis de vulnerabilidad, y su fin primordial es definir las acciones a seguir en cualquier situación de emergencia o de desastre.

En el marco del desaparecido Sistema Nacional de Defensa Civil - SINADECI ${ }^{i i i}$, regulado por el Decreto Ley N 19338 y reglamentado por el Decreto Supremo Nº05-88-SGMD, se definía al Plan de Operaciones de Emergencia como un plan operativo que organiza la preparación y la respuesta a la emergencia, considerando los riesgos del área bajo su responsabilidad y los medios disponibles en el momento. Este plan es evaluado periódicamente mediante simulaciones y simulacros.

La norma ISO 22301 define el "plan de continuidad de negocio" (plan de continuidad operativa) como los procedimientos documentados que guian a la organización a responder, recuperar, reanudar y restablecer a niveles predefinidos después de una alteración.

En el documento Lineamientos para la Gestión de Continuidad Operativa de la Entidades Públicas en los Tres Niveles de Gobierno se define el "Plan de Continuidad Operativa" como el instrumento que debe formar parte de las operaciones habituales de la Entidad, que incluye la identificación de las actividades y servicios críticos que requieran ser ejecutados y prestados de manera ininterrumpida, la determinación de las medidas y acciones que permitan que la entidad de manera eficiente y eficaz siga cumpliendo con su objetivos, así como la relación del personal que se encontrará a cargo de la ejecución de las mencionadas actividades. Incluye protocolos, la realización de pruebas y ensayos, entre otros elementos señalados en dicho documento.

Por su lado, De la Torre ${ }^{4}$ define al Plan de Continuidad Operativa como el documento que está enfocado a asegurar la continuidad del negocio, cuando de repente ocurre un incidente inesperado; este plan lo que intenta es no detener la productividad de la empresa, e intentar que la situación que ha sucedido en ese momento nos afecte lo menos posible.

La organización RedInfo ${ }^{5}$ señala que el objetivo del Plan de Continuidad Operativa es establecer los procedimientos y recursos necesarios para la administración de procesos críticos en situación de contingencia. Además, define el personal y los cargos claves que deben asumir en la toma de decisiones en caso de contingencias.

Se define, en el Reglamento de la Ley N²9664 - Ley del Sistema Nacional de Gestión de Riesgo de Desastre y en el documento Lineamientos para la Formulación y Aprobación de Planes de Contingencia, aprobado mediante Resolución Ministerial $\mathrm{N}^{\circ}$ 188-2015-PCM, al Plan de Contingencia como los procedimientos específicos preestablecidos de coordinación, alerta, movilización y respuesta ante una ocurrencia o inminencia de un evento particular para el cual se tiene escenarios definidos.

De la Torre ${ }^{4}$ indica que un Plan de Contingencia consiste en restar el impacto financiero que puede causar un "incidente" inesperado en la compañía dentro del marco de los procedimientos habituales

\footnotetext{
iii EI SINADECI fue reemplazado por el Sistema Nacional de Gestión de Riesgos de Desastres.

iiii Estado de daños sobre la vida, el patrimonio y el medio ambiente ocasionados por la ocurrencia de un fenómeno natural o inducido por la acción humana que alterna el normal desenvolvimiento de las actividades de la zona afectada (Decreto Supremo No 048-2011-PCM).
}

de la empresa, este plan trabaja para recuperar a la compañía de los imprevistos especiales que se puedan dar, y que por su causa interrumpen el sistema de producción.

Según lo señala Borghello ${ }^{6}$, un plan de continuidad incluye un plan de contingencias debido que este último es aplicado únicamente cuando se produjo un desastre mientras que el plan de continuidad aparte de tener medidas en caso de desastre también contempla el análisis de las vulnerabilidades y desarrolla contramedidas para mitigar dichas vulnerabilidades. Por tanto, el plan de continuidad no solo es correctivo como el plan de contingencias, sino que también es preventivo.

Sin embargo, hay que considerar también que el Servicio Nacional de Aprendizaje - SENA ${ }^{3}$, establece que los planes de contingencia son planes de emergencia específicos, destinados a la prevención y control de amenazas particulares identificadas por áreas de trabajo o por empresa.

Por lo expuesto, se plantea las siguientes definiciones a ser tomada en cuenta al desarrollo de la investigación:

- El Plan de Emergencia tiene un enfoque de prevención y control de las potenciales situaciones de emergencia que pudieran acontecer a una organización. ${ }^{\text {iii }}$

- El Plan de Continuidad Operativa tiene un enfoque preventivo que establece acciones para hacer frente a la ocurrencia de un evento inesperado con el objetivo de evitar la paralización de las operaciones, además contempla las acciones de contingencia de respuesta inmediata para emergencias externas de bajo riesgo.

- El Plan de Contingencia tiene un enfoque reactivo que establece acciones para hacer frente a la ocurrencia de un evento inesperado que provocaron la paralización de las operaciones.

El presente estudio se planteó el objetivo de establecer la estructura del Plan de Continuidad Operativa para una Empresa de Saneamiento de Agua, a partir de los resultados del diagnóstico documentario sobre los lineamientos y prácticas de la Gestión de Continuidad Operativa. Para ello se plantearon las siguientes acciones:

- Desarrollar un diagnóstico documentario sobre los lineamientos y prácticas de la Gestión de Continuidad Operativa a través de la búsqueda de normas técnicas, investigaciones y artículos especializados que aborden el tema de la Gestión de la Continuidad Operativa asociada a la Gestión de Riesgos de Desastres.

- Consolidar y procesar la información técnica para la definición de términos, identificación de buenas prácticas y determinación de lineamientos para la formulación del Plan de Continuidad Operativa.

- Definir los elementos de la estructura del Plan de Continuidad Operativa.

\section{MATERIAL Y MÉTODOS}

\section{Empresa de Estudio}

La investigación tomó como objeto de estudio a una empresa pública de servicio de saneamiento de agua cuyos roles y competencias están establecidos en el Decreto Legislativo $\mathrm{N}^{\circ} 1280$ (promulgado en diciembre de 2016), mediante el cual se aprobó la Ley Marco de Gestión y Prestación de Servicios de Saneamiento.

Para agosto 2017, se calculaba que la población atendida por esta empresa era de 11,18 millones de habitantes asentados en 50 distritos. 
Tabla 2.

Normas Internacionales relacionadas a la Continuidad Operativa.

\begin{tabular}{|c|c|}
\hline Norma Técnica & Descripción \\
\hline \multirow[t]{2}{*}{ Estándar DRI } & $\begin{array}{l}\text { La certificación del estándar internacional DRI reconoce las prácticas } \\
\text { profesionales para la Gestión de Continuidad de Negocio. }\end{array}$ \\
\hline & $\begin{array}{l}\text { Plantea que el objetivo de la continuidad de negocio es permitir a las } \\
\text { operaciones comerciales de la empresa seguir operando bajo } \\
\text { condiciones adversas, al implantar estrategias adecuadas, objetivos } \\
\text { de recuperación, planes de gestión de crisis y estrategias de gestión } \\
\text { de riesgos. }\end{array}$ \\
\hline \multirow[t]{2}{*}{$\begin{array}{l}\text { Norma BS } \\
25999\end{array}$} & $\begin{array}{l}\text { La norma BSI } 25999 \text { plantea que la gerencia del plan de continuidad } \\
\text { del negocio es un proceso que abarca los riesgos y sus potenciales } \\
\text { impactos en los procesos de la organización. }\end{array}$ \\
\hline & $\begin{array}{l}\text { Además, promueve una estructura para mantener la flexibilidad y } \\
\text { continuidad de los procesos organizacionales basándose a la norma. }\end{array}$ \\
\hline $\begin{array}{l}\text { Norma ISO } \\
22399\end{array}$ & $\begin{array}{l}\text { La norma ISO } 22399 \text { establece que el plan de continuidad presenta } \\
\text { principios y elementos generales para la preparación en caso de un } \\
\text { incidente y tener la continuidad operativa de la organización. }\end{array}$ \\
\hline $\begin{array}{l}\text { Norma ISO } \\
27001\end{array}$ & $\begin{array}{l}\text { La norma ISO } 27001 \text { define los requisitos para un sistema de gestión } \\
\text { de la seguridad de la Información, permitiendo a la organización } \\
\text { definir procesos para establecer, implantar, operar, supervisar, revisar, } \\
\text { mantener y mejorar el sistema de gestión desarrollado. }\end{array}$ \\
\hline \multirow[t]{2}{*}{ ITDR } & $\begin{array}{l}\text { El estándar Information Technology Disaster Recovery - ITDR trata } \\
\text { sobre la ejecución de los procesos críticos de información y } \\
\text { tecnología de comunicaciones. }\end{array}$ \\
\hline & $\begin{array}{l}\text { Su finalidad es apoyar a las organizaciones implementar una } \\
\text { correcta estrategia para servicios de información y tecnología } \\
\text { de comunicaciones. }\end{array}$ \\
\hline \multirow[t]{3}{*}{ BS 25777} & $\begin{array}{l}\text { La finalidad del BS } 25777 \text { es la implementación de una correcta } \\
\text { estrategia para servicios de información y tecnologia de } \\
\text { comunicaciones. }\end{array}$ \\
\hline & $\begin{array}{l}\text { También conocido como Information and Communication } \\
\text { Technology - ICT, cuenta con algunos elementos que permiten su } \\
\text { desarrollo de una mejor manera. }\end{array}$ \\
\hline & $\begin{array}{l}\text { Este estándar está alineado al BS } 25999-1 \text {, enfocado a los procesos } \\
\text { del negocio. }\end{array}$ \\
\hline ITIL & $\begin{array}{l}\text { La Biblioteca de Infraestructuras de Tecnologias de la Información } \\
\text { (ITIL) contiene como punto fundamental un Modelo Plan de } \\
\text { Continuidad de Negocios, con los siguientes objetivos: } \\
\text { 1. Garantizar la pronta recuperación de los servicios de tecnología } \\
\text { de información tras un desastre. } \\
\text { 2. Establecer políticas y procedimientos que eviten, en la medida } \\
\text { posible, las perniciosas consecuencias de un desastre o causa } \\
\text { de fuerza mayor. }\end{array}$ \\
\hline \multirow[t]{2}{*}{ NFPA 1600} & $\begin{array}{l}\text { La norma NFPA } 1600 \text { brinda los lineamientos para abordar la } \\
\text { Continuidad de Negocio y cada una de sus fases, para la efectiva } \\
\text { Administración de Continuidad de Negocio. Esta norma puede servir } \\
\text { para reforzar el Plan de Continuidad de Negocio, ya que esta está } \\
\text { enfocada al tema de seguridad de los empleados y de la misma } \\
\text { organización. }\end{array}$ \\
\hline & $\begin{array}{l}\text { Algunas de las fases pueden ayudar a fortalecer las fases de } \\
\text { comunicación entre empleados en un escenario y administración } \\
\text { de incidentes. }\end{array}$ \\
\hline \multirow[t]{2}{*}{ ISO 27002} & $\begin{array}{l}\text { La norma ISO } 27002 \text { hace referencia a un código de prácticas para } \\
\text { la seguridad de la información. }\end{array}$ \\
\hline & $\begin{array}{l}\text { Además, de ser enfocado mayormente a la información por lo que } \\
\text { puede ser de gran ayuda al momento de tomar en cuenta la } \\
\text { continuidad de la seguridad que la información debe tener. }\end{array}$ \\
\hline \multirow[t]{3}{*}{ ISO 22301} & $\begin{array}{l}\text { La norma ISO } 22301 \text { tiene como referencia la gestión de la } \\
\text { continuidad de negocio y ha sido desarrollada para ayudar a las } \\
\text { organizaciones a minimizar el riesgo de sufrir interrupciones. }\end{array}$ \\
\hline & Esta norma no es tan diferente a la BS 25999. \\
\hline & $\begin{array}{l}\text { Es preciso resaltar que se aborda el análisis del impacto y } \\
\text { la estrategia }\end{array}$ \\
\hline
\end{tabular}

En ese sentido para junio de 2016, la empresa alcanzó una cobertura de 93,4\% en el servicio de agua potable y, 90,3\% en el servicio de alcantarillado.

\section{Revisión de Documentación relacionada a la Continuidad Operativa}

Para la revisión de la documentación normativa, se tomó en consideración lo desarrollado por Alexander, UrbiCAD y Sáez ${ }^{7-9}$, para la identificación de las normas técnicas internacionales que guardan relación con la gestión de la continuidad operativa. Cabe precisar que la totalidad de las normas consultadas están vinculadas a la gestión de la continuidad operativa, pero desde la perspectiva de la tecnología de la información, a excepción de la norma NFPA 1600 Programas de Manejo de Desastres/Emergencias y Continuidad de Negocios cuya orientación se da en la respuesta a incidentes que puedan afectar a las personas, propiedad y medio ambiente.

Para la revisión de la documentación técnica se identificó y consolidó las publicaciones obtenidas de investigaciones, revistas técnicas y foros especializados; sin embargo, como se mencionó en lo correspondiente a la documentación normativa, estas publicaciones han sido desarrolladas en el ámbito de las tecnologías de la información. Con la revisión de documentos benchmark (documentos modelo de diversas organizaciones), se buscó establecer los lineamientos y elementos comunes para la determinación de la estructura del Plan de Continuidad Operativa.

\section{RESULTADOS}

\section{Estructura del Plan de Continuidad Operativa}

A partir de los resultados de la revisión de la documentación, se estableció la estructura del Plan de Continuidad Operativa bajo la premisa que este le permita responder ante una amenaza que pueda paralizar sus operaciones. En la siguiente tabla se presenta la descripción de cada uno de los elementos de la estructura del Plan de Continuidad Operativa.

\section{Estado Situacional de la Empresa en Estudio}

En la empresa de estudio se efectuó un Análisis de Impacto de Negocio (BIA) - Estratégico que comprendió un universo de trece (13) macro procesos (Nivel 0), conformados por 169 procesos de Nivel 1, determinándose seis (6) macroprocesos con 92 procesos de Nivel 1 con prioridad de recuperación "Urgente" tomando en cuenta el criterio MTPD ${ }^{\text {iiiii }}<=2$ meses.

Además, se efectuó un BIA - Operativo a los 6 macroprocesos, identificándose 43 procesos de Nivel 1 que deberían ser priorizados tomando como base el mismo criterio de selección que se usó para evaluar los macroprocesos: MTPD tuviera un valor menor o igual a dos (02) meses.

Como parte del Análisis de Impacto en el Negocio, se concluyó que los macroprocesos relacionados con la "Producción y Distribución de Agua Potable" y "Recolección y Tratamiento de Aguas Residuales" son los más críticos (MTPD $<=1$ semana) debido a que ante una interrupción de los mismos se afectaría directamente a la población atendida por la empresa en estudio.

Por tanto, si bien en el BIA Operativo se identificó 25 procesos de Nivel $1^{\text {iiiiii }}$; se propone priorizar 17 procesos que corresponden

\footnotetext{
iiiii Máximo periodo tolerable de interrupción (MTPD, siglas en inglés).

iiiiii Los procesos nivel 1 priorizados son 25; sin embargo, Deloitte ha desplegado los procesos por sede dando un total de 43 procesos.
} 
Tabla 3.

Publicaciones relacionadas a la Continuidad Operativa.

\begin{tabular}{|c|c|}
\hline Tópico & Descripción \\
\hline $\begin{array}{l}\text { Plan de } \\
\text { Contingencia }\end{array}$ & $\begin{array}{l}\text { El Foro de Profesionales Latinoamericanos de Seguridad }{ }^{10} \text { hace } \\
\text { referencia a la función primordial de un Plan de Contingencia, } \\
\text { entendiéndose como los procedimientos alternativos al orden normal } \\
\text { de una empresa, cuyo fin es permitir el normal funcionamiento aun } \\
\text { cuando una de sus funciones se viera dañada por un factor interno o } \\
\text { externo. Dentro de su función principal de un Plan de Contingencia } \\
\text { es la continuidad de las operaciones de la empresa, donde se dividen } \\
\text { en cuatro etapas: } \\
\text { 1. Evaluación, } \\
\text { 2. Planificación, } \\
\text { 3. Pruebas de viabilidad, y } \\
\text { 4. Ejecución. } \\
\text { Positiva Compañia de Seguros }{ }^{11} \text { hace referencia a un Plan de } \\
\text { Emergencias y Contingencias, como herramienta administrativa y } \\
\text { operativa, cuyo propósito es dar respuesta ante una eventualidad } \\
\text { que se pueda presentar. } \\
\text { Primero se requiere definir el alcance, asi como conocer el } \\
\text { propósito de la organización. Esta etapa comprende el análisis de } \\
\text { riesgo y la clasificación de amenazas junto con las acciones a tomar. } \\
\text { Por otro lado, muestra un diseño de estructuración para asignar } \\
\text { funciones y responsabilidades, además abarca los planes de acción, } \\
\text { definiendo cada procedimiento a desarrollar según los recursos, } \\
\text { suministros a utilizar. }\end{array}$ \\
\hline $\begin{array}{l}\text { Plan de } \\
\text { Continuidad } \\
\text { de Negocios }\end{array}$ & $\begin{array}{l}\text { Sáez }{ }^{9} \text { hace una propuesta metodológica para la implementación de } \\
\text { un Plan de Continuidad de Negocio. } \\
\text { Dentro de ello, se incluye las metodologias existentes y estándares } \\
\text { internacionales para su implementación; además, identifica las } \\
\text { actividades necesarias para diseñar, implementar y mantener el } \\
\text { Plan de Continuidad Operativa; y analiza la cultura organizacional } \\
\text { de la empresa al momento de su implementación. } \\
\text { Cabe destacar que desarrolla matrices de nivel de riesgo, también da a } \\
\text { conocer las funciones principales de cada norma según su metodología. } \\
\text { Contempla la toma de medidas preventivas en caso de incidentes al } \\
\text { no ser gestionadas adecuadamente. } \\
\text { Así mismo, presenta estrategias de recuperación con el fin de } \\
\text { detectar las actividades criticas de la organización. } \\
\text { Ferrer } \\
\text { del } \text { hace referencia a una metodología para la implementación } \\
\text { procedimientos e información, además de contar con una metodología } \\
\text { que comprende desde la politica hasta el mantenimiento del plan } \\
\text { conjuntamente agregando cuadros de referencias. }\end{array}$ \\
\hline $\begin{array}{l}\text { Plan de } \\
\text { Continuidad } \\
\text { Operativa }\end{array}$ & $\begin{array}{l}\text { Flavio }{ }^{13} \text { señala que el Plan de Continuidad Operativa busca la } \\
\text { continuidad y restablecimientos de los procesos. Dentro de sus } \\
\text { estrategias se cuenta los criterios de recuperación según sus } \\
\text { objetivos de cada recurso crítico. }\end{array}$ \\
\hline $\begin{array}{l}\text { Plan de } \\
\text { Contingencias } \\
\text { y Continuidad } \\
\text { de Negocio }\end{array}$ & $\begin{array}{l}\text { El Instituto Nacional de Ciberseguridad-INCIBE14 hace referencia a } \\
\text { una serie de pasos para una implementación de Plan de Continuidad } \\
\text { de Negocios en una empresa, viendo así los factores y beneficios que } \\
\text { este pueda dar. }\end{array}$ \\
\hline $\begin{array}{l}\text { Planes de } \\
\text { emergencia y } \\
\text { contingencia }\end{array}$ & $\begin{array}{l}\text { La Dirección de Prevención y Atención de Emergencias de } \\
\text { Colombia15 propone la siguiente estructura para los planes de } \\
\text { emergencia y contingencia: } \\
\text { - Introducción } \\
\text { - Justificación } \\
\text { - Objetivos } \\
\text { - Alcance } \\
\text { - Información general } \\
\text { - Análisis de riesgo } \\
\text { - Esquema organizacional para la atención de emergencias } \\
\text { - Planes de acción } \\
\text { - Análisis de suministros, servicios y recursos } \\
\text { - Planes de contingencias } \\
\text { - Programa de capacitación } \\
\text { - Implementación } \\
\text { - Auditoría } \\
\text { - Actualización } \\
\text { - Anexos }\end{array}$ \\
\hline
\end{tabular}

Tabla 4.

Documentos Modelos relacionados a Continuidad Operativa.

\begin{tabular}{|c|c|c|}
\hline $\begin{array}{l}\text { Tipo de } \\
\text { Organización }\end{array}$ & Documento & Descripción del Documento \\
\hline $\begin{array}{l}\text { Empresa de } \\
\text { transporte } \\
\text { de carga }\end{array}$ & $\begin{array}{l}\text { Plan de } \\
\text { Contingencia } \\
\text { de Continuidad } \\
\text { de Negocio }\end{array}$ & $\begin{array}{l}\text { El documento tiene como objetivo establecer las } \\
\text { diferentes pautas y acciones que permitan minimizar los } \\
\text { posibles riesgos ante una eventualidad que pueda } \\
\text { significar una paralización o falta de continuidad del servicio. } \\
\text { La estructura de este documento contempla: } \\
\text { - Concepto } \\
\text { - Antecedentes } \\
\text { - Objetivos } \\
\text { - Tiempo de recuperación } \\
\text { - Alcance } \\
\text { - Procedimientos } \\
\text { - Conclusiones. }\end{array}$ \\
\hline \multirow[t]{2}{*}{$\begin{array}{l}\text { Empresa de } \\
\text { servicio de } \\
\text { mensajería } \\
\text { (courrier) }\end{array}$} & $\begin{array}{l}\text { Plan de } \\
\text { Gestión de } \\
\text { Riesgos y } \\
\text { Continuidad } \\
\text { del Negocio }\end{array}$ & $\begin{array}{l}\text { El documento contiene las medidas técnicas, humanas y } \\
\text { organizativas para garantizar la continuidad de las } \\
\text { operaciones. } \\
\text { La estructura de este documento contempla: } \\
\text { - Información general } \\
\text { - Objetivos } \\
\text { - Organización } \\
\text { - Identificación del plan } \\
\text { - Plan de monitoreo / control del riesgo } \\
\text { - Procedimiento general } \\
\text { - Procedimiento detallado } \\
\text { - Manejo y control del riesgo } \\
\text { - Alcance } \\
\text { - Implantación. }\end{array}$ \\
\hline & $\begin{array}{l}\text { Matriz de } \\
\text { Riesgos } \\
\text { Operacionales }\end{array}$ & $\begin{array}{l}\text { La estructura de la Matriz de Riesgos Operacionales } \\
\text { contempla. } \\
\text { - Riesgos críticos operacionales } \\
\text { - Consecuencias } \\
\text { - Causas } \\
\text { - Impacto } \\
\text { - Probabilidad } \\
\text { - Severidad } \\
\text { - Nivel de riesgo } \\
\text { - Valoración del riesgo } \\
\text { - Plan de control preventivo } \\
\text { - Plan de respuesta } \\
\text { - Tiempo }\end{array}$ \\
\hline Banco & $\begin{array}{l}\text { Plan de } \\
\text { Continuidad y } \\
\text { Manejo de } \\
\text { Crisis ante } \\
\text { Desastres }\end{array}$ & $\begin{array}{l}\text { Este es un documento parte de una presentación del año } \\
2013 \text {, de donde se resalta la matriz de evaluación de } \\
\text { servicios ante un desastre. De acuerdo a lo presentado, } \\
\text { este documento puede ser considerado como un plan de } \\
\text { contingencias. }\end{array}$ \\
\hline Ministerio & $\begin{array}{l}\text { Plan de } \\
\text { Continuidad } \\
\text { Operativa }\end{array}$ & $\begin{array}{l}\text { Se resalta de este documento su estructura: } \\
\text { - Introducción } \\
\text { - Finalidad } \\
\text { - Objetivos } \\
\text { - Base legal } \\
\text { - Ámbito de aplicación } \\
\text { - Contenido } \\
\text { - Acciones previstas para la gestión } \\
\text { - de continuidad operativa } \\
\text { - Rinanciamiento y presupuesto } \\
\text { - Anexomendaciones básicas }\end{array}$ \\
\hline $\begin{array}{l}\text { Entidad } \\
\text { Estatal de } \\
\text { Recreación } \\
\text { y Deporte }\end{array}$ & $\begin{array}{l}\text { Plan de } \\
\text { Emergencia y } \\
\text { Contingencias }\end{array}$ & $\begin{array}{l}\text { En este documento se presenta un análisis de } \\
\text { vulnerabilidad de los sistemas y procesos que incluye } \\
\text { dentro de los criterios de evaluación la disponibilidad } \\
\text { de un plan de continuidad de negocios. }\end{array}$ \\
\hline
\end{tabular}

Fuente: Elaboración Propia. 
a los macroprocesos críticos Producción y Distribución de Agua Potable y Recolección y Tratamiento de Aguas Residuales, en términos de MTPD, RTO ${ }^{\text {iiiiiii }}$ y RPO Riiiiiiii $^{\text {in }}$

\section{Elaboración del Plan de Continuidad Operativa}

Se identificaron las amenazas con riesgos de tipo operativos en las diferentes actividades las cuales son ejecutados por los diferentes equipos de trabajos para brindar un adecuado servicio de

\section{Tabla 5.}

Estructura del Plan de Continuidad Operativa.

$\begin{array}{ll}\text { Estructura } & \text { Descripción } \\ \text { I. } & \text { Este es el primer elemento del cuerpo o texto del documento, en el } \\ \text { cual se presenta y señala la importancia y orientación del } \\ \text { Plan de Continuidad Operativa } \\ \text { (PCO), los antecedentes, los objetivos, el significado que el PCO } \\ \text { tiene en el avance del campo respectivo y la aplicación. } \\ \text { Además, se mencionan los alcances, las limitaciones y la } \\ \text { metodología empleada. } \\ \text { II. } \\ \text { Se presenta información sobre la organización. } \\ \text { A. Estado Situacional: } \\ \text { Comprende información general de la organización, que incluye: } \\ \text { actividad económica, organización, politicas de gestión, etc. } \\ \text { B. Procesos } \\ \text { Se incluye la interacción de los macroprocesos de la organización } \\ \text { (Mapa de Procesos). } \\ \text { C. Instalaciones } \\ \text { Se presenta la información de las sedes administrativas y } \\ \text { comerciales, plantas de tratamiento y otras. Esta información } \\ \text { estará soportada con planos o diagramas que indiquen y faciliten } \\ \text { la ubicación de las instalaciones. } \\ \text { D. Roles y Responsabilidades } \\ \text { Es la asignación de los roles dentro de la estructura funcional de } \\ \text { la organización para la aprobación, implementación, mantenimiento } \\ \text { y mejora del PCO. }\end{array}$

III. Se lista la normativa legal aplicable a la gestión de continuidad Marco Legal operativa, asi como la normativa técnica tomada como referencia.

IV.

Alcance

V.

Objetivos

Vl. Definiciones

VII.

Identificación de Amenazas

VIII. Acciones para continuidad operativa agua potable y alcantarillado. En la siguiente tabla, se presenta las amenazas del Macroproceso "Producción y Distribución de Agua Potable" - Proceso "Producción de agua superficial". A partir de esto, se procede a la determinación de las acciones para la continuidad operativa para cada proceso:

iiiiii Tiempo objetivo de recuperación (RTO, por sus siglas en inglés).

iiiiiii Punto objetivo de recuperación (RPO, por sus siglas en inglés).

\section{Estructura Descripción}

Por lo tanto, el flujo es indispensable para la comunicación efectiva y las posibles respuestas que se puedan emitir frente a una amenaza, sin dejar de lado u omitir tomar alguna acción inmediata.

La activación del PCO permitirá medir los parámetros (principalmente de tiempo de respuesta) en que los Responsables de Area responden frente a las amenazas asociadas a los riesgos operacionales para aplicar los protocolos de respuesta u otras acciones pertinentes.

B. Ejecución

Bajo la responsabilidad de los Jefes de Área, comprende la aplicación de los protocolos de respuesta, que incluye la asignación de recursos necesarios que deben movilizarse, según corresponda. Se entiende como protocolo de respuesta al conjunto de acciones y reglas que se aplican de acuerdo a la amenaza que se presenta. Los protocolos de respuesta incluyen:

- La amenaza asociada a los riesgos operacionales.

- Las acciones y reglas que se aplican frente a las amenazas para evitar la paralización de las operaciones. En caso de que la paralización de las operaciones es inminente o se haya producido la paralización, se vinculará a los Planes de Contingencia / Emergencia que se hayan desarrollado para tal fin (Gestión de Crisis).

- Los responsables son aquellos puestos asignados para la aplicación de las acciones y reglas. A este nivel corresponde establecer la Línea de Sucesión (responsable titular y responsables alternos) y la Cadena de Mando (tomadores de decisiones).

- Los plazos de tiempo en que se deben desarrollar y tomar acciones y reglas frente a la ocurrencia de las amenazas.

IX.

Comprende el diseño de la programación de actividades específicas para

Difusión e la difusión del PCO, asi como su implementación proyectada a corto, implementación mediano o largo plazo.

del Plan La programación incluye una serie de actividades necesarias para su

Continuidad implementación; algunas de ellas, son:

Operativa - Acciones de divulgación del plan, en todos los niveles de la organización.

- Definición del plan de capacitación.

- Actividades de seguimiento, evaluación y ajustes del PCO.

X. La evaluación del desempeño del PCO comprende las pruebas y

Evaluación de ensayos, para ello se diseña el programa de simulación y simulacros desempeño para poner a prueba el PCO que permita identificar fortalezas y oportunidades de mejora.

XI. El proceso de mejora del PCO debe efectuarse mediante el sistema de Mejora control de cambios del Sistema de Gestión Integrado de la organización, que permita conocer el progreso y los cambios realizados al $\mathrm{PCO}$, de acuerdo con las evaluaciones desarrolladas y sus actividades.

Se debe asignar a un responsable para el seguimiento al PCO, quien periódicamente informará a la instancia pertinente de la organización las actividades ejecutadas y los diferentes aspectos de modificación y control del PCO.

XII. Comprende a todos aquellos documentos, formatos, planos, mapas, Anexos diagramas de procesos, entre otros elementos, que complementan el cuerpo del PCO y que se relacionan, directa o indirectamente, con el mismo. 
Tabla 6.

\begin{tabular}{c|c} 
Macroproceso & Tipo \\
\hline $\begin{array}{c}\text { Recolectar, tratar y disponer } \\
\text { aguas residuales }\end{array}$ & Realización del producto \\
\hline $\begin{array}{c}\text { Producción y abastecimiento } \\
\text { de agua potable }\end{array}$ & Realización del producto \\
\hline Comercializar & Realización del producto \\
\hline $\begin{array}{c}\text { Administrar los sistemas de } \\
\text { tecnologias de información }\end{array}$ & Gestión de recursos \\
\hline Administrar las finanzas y contabilidad & Gestión de recursos \\
\hline Administrar y desarrollar \\
los recursos humanos
\end{tabular}

Fuente: Elaboración Propia.

El Plan de Continuidad Operativa contempla un programa de difusión e implementación que incluye una serie de actividades necesarias; algunas de ellas son:

- Acciones de divulgación del plan, en todos los niveles de la organización.

- Desarrollo de ejercicios de simulación y simulacros, periódicamente.

- Adquisición de recursos y elementos complementarios.

- Elaboración de la base de datos y actualización periódica de la misma.

- Definir el Plan de capacitación.

- Actividades de seguimiento, evaluación y ajustes del plan.

Además, para la evaluación del desempeño del Plan de Continuidad Operativa debe considerarse, entre otras, las siguientes prácticas:

- Simulacros: practica y prueba la efectividad de tu plan.

- Reiniciar operaciones: practica cómo sería el reinicio de actividades.

Es recomendable que se realice los ejercicios que se consideren necesarios y realistas. Se puede incrementar los niveles de complejidad de los ejercicios de prueba y adaptarse a los diferentes escenarios para mejorar las capacidades de continuidad operativa.

\section{DISCUSIÓN}

En el Estado Peruano, las Entidades Públicas en todos los niveles de gobierno deben implementar de la Gestión de la Continuidad Operativa con el propósito de contribuir con el Sistema Nacional de Gestión de Riesgo de Desastres (SINAGERD). Esta obligación contempla la elaboración de un Plan de Continuidad Operativa; sin embargo, no se ha identificado documentación normativa, técnica ni de benchmark que establezca la estructura del contenido de dicho documento.

Existe abundante documentación asociada a la gestión de la continuidad operativa pero bajo el enfoque de la gestión de la tecnología de la información. No se descarta que dicha documentación pueda ser aplicable a la empresa en estudio, pero sólo en lo que respecta a sus sistemas de informáticos y bases de datos, mas no a la continuidad de las operaciones de sus macroprocesos.

\section{Con esta investigación, se concluye que:}

- El Plan de Continuidad Operativa tendrá como objetivo esta-
Tabla 7.

\begin{tabular}{|c|c|c|c|}
\hline \multicolumn{2}{|c|}{ Macroproceso } & \multicolumn{2}{|c|}{$\begin{array}{c}\text { Producción y Distribución } \\
\text { de Agua Potable }\end{array}$} \\
\hline \multicolumn{2}{|c|}{ Proceso Nivel 1} & \multicolumn{2}{|c|}{ Producción de Agua Superficial } \\
\hline $\begin{array}{l}\text { SubProceso } \\
\text { Captación de } \\
\text { Agua }\end{array}$ & 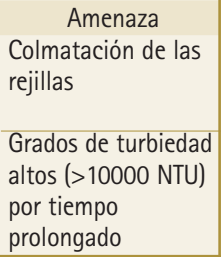 & $\begin{array}{l}\quad \text { Causa } \\
\text { Cantidad de } \\
\text { residuos sólidos en } \\
\text { la fuente de agua } \\
\text { Huaycos }\end{array}$ & $\begin{array}{l}\quad \text { Impacto } \\
\text { Limita la captación } \\
\text { de agua }\end{array}$ \\
\hline Desarenación & $\begin{array}{l}\text { Falta de disponibili- } \\
\text { dad de Polímero } \\
\text { aniónico en épocas } \\
\text { de avenida } \\
\text { (diciembre-abril) o } \\
\text { estiaje (mayo- } \\
\text { noviembre) }\end{array}$ & $\begin{array}{l}\text { Captación de aguas } \\
\text { con altas turbidades } \\
\text { que demanden un } \\
\text { mayor consumo del } \\
\text { insumo y } \\
\text { disminuyan } \\
\text { el stock }\end{array}$ & $\begin{array}{l}\text { Limita la captación de } \\
\text { aguas si la turbidad es } \\
>800 \text { NTU. Por lo } \\
\text { general, en épocas de } \\
\text { avenida (diciembre- } \\
\text { abril) }\end{array}$ \\
\hline Pre cloración & $\begin{array}{l}\text { Falta de corriente } \\
\text { eléctrica }\end{array}$ & $\begin{array}{l}\text { Obstrucción en la } \\
\text { succión de la } \\
\text { bomba de agua. } \\
\text { Fallas propias en las } \\
\text { bombas de agua } \\
\text { Cortes programados } \\
\text { o intempestivos de } \\
\text { la empresa } \\
\text { eléctrica. }\end{array}$ & $\begin{array}{l}\text { Contaminación } \\
\text { biológica en la fuente. } \\
\text { Disminución de cloro } \\
\text { residual en la planta. } \\
\text { Incumplimientos } \\
\text { legales. }\end{array}$ \\
\hline $\begin{array}{l}\text { Coagulación / } \\
\text { Floculación }\end{array}$ & $\begin{array}{l}\text { Falla de bombas } \\
\text { dosificadoras } \\
\text { Falta de } \\
\text { disponibilidad } \\
\text { de insumos }\end{array}$ & $\begin{array}{l}\text { Fallas propias en las } \\
\text { bombas de agua. } \\
\text { Incidentes en el } \\
\text { proceso de } \\
\text { adquisición del } \\
\text { polímero. } \\
\end{array}$ & $\begin{array}{l}\text { Incremento de } \\
\text { la turbiedad. }\end{array}$ \\
\hline Post cloración & $\begin{array}{l}\text { Fallas en el sistema } \\
\text { de post cloración }\end{array}$ & $\begin{array}{l}\text { Fallas propias en las } \\
\text { bombas de agua. }\end{array}$ & $\begin{array}{l}\text { Incumplimiento de } \\
\text { rangos de cloro }\end{array}$ \\
\hline & & & $\begin{array}{l}\text { residual }(0.7-1.7 \mathrm{mg} / \mathrm{l}) \\
\text { No cumplir con los } \\
\text { LMP requeridos } \\
\text { (Bacterias heterotró- } \\
\text { ficas: } 500 \mathrm{ufc} / \mathrm{ml} \text { ) }\end{array}$ \\
\hline & $\begin{array}{l}\text { Falla de Corriente } \\
\text { Eléctrica }\end{array}$ & $\begin{array}{l}\text { Cortes programados } \\
0 \text { intempestivos de } \\
\text { la empresa eléctrica. }\end{array}$ & $\begin{array}{l}\text { Disminución de cloro } \\
\text { residual en la planta. } \\
\text { Incumplimientos } \\
\text { legales. }\end{array}$ \\
\hline
\end{tabular}

blecer las acciones para hacer frente a la ocurrencia de amenazas asociadas a los riesgos operacionales que puedan paralizar las operaciones.

- La estructura del Plan de Continuidad Operativa debe comprender 12 puntos: Introducción, Generalidades, Marco Legal, Alcance, Objetivos, Definiciones, Identificación de Amenaza. Acciones para la continuidad operativa, Difusión e implementación del Plan de Continuidad Operativa, Evaluación de desempeño, Mejora y Anexos.

- Las acciones para la continuidad operativa contemplan el desarrollo de protocolos de respuesta con un enfoque preventivo que comprenden la identificación de las amenazas asociada a los riesgos operativos, las acciones y reglas, responsables (incluye línea de sucesión y cadena de Mando), y plazos; además incluyen las acciones de contingencia de respuesta inmediata para emergencias externas de bajo riesgo.

- La evaluación de desempeño del Plan de Continuidad Operativa comprende las pruebas y ensayos que permitirán identificar fortalezas y oportunidades de mejora. 


\section{Tabla 8.}

Acciones para continuidad operativa para la Producción de Agua Potable Superficial.

\begin{tabular}{|c|c|c|}
\hline \multicolumn{2}{|l|}{ Macroproceso } & Producción y Distribución de Agua Potable \\
\hline \multicolumn{2}{|l|}{ Proceso Nivel 1} & Producción de Agua Superficial \\
\hline \multirow[t]{2}{*}{ Amenaza } & Acciones Preventivas & Acciones de Contingencia \\
\hline & Subproceso: Captación de agua & \\
\hline Colmatación de las rejillas & $\begin{array}{l}\text { Se dispone de personal para realizar la limpieza } \\
\text { de rejillas y retirar los materiales sólidos. }\end{array}$ & Incrementar personal para realizar la limpieza de rejillas. \\
\hline \multirow[t]{2}{*}{$\begin{array}{l}\text { Grados de turbiedad altos (>10 } 000 \text { NTU) } \\
\text { por tiempos prolongados }\end{array}$} & $\begin{array}{l}\text { Se monitorea constantemente los grados } \\
\text { de turbidez de la fuente. }\end{array}$ & $\begin{array}{l}\text { Apertura de compuertas radiales para evitar la captación } \\
\text { de aguas hasta que bajen los niveles de turbiedad } \\
\text { a } 10000 \text { NTU. }\end{array}$ \\
\hline & Subproceso: Desarenado & \\
\hline \multirow[t]{2}{*}{$\begin{array}{l}\text { Falta de disponibilidad de polímero aniónico en } \\
\text { época de avenida (dic-abr) o estiaje (may-nov) }\end{array}$} & $\begin{array}{l}\text { Llevar el control del stock del insumo. } \\
\text { Adquirir insumo para dos (02) años. }\end{array}$ & Compra de emergencia. \\
\hline & Subproceso: Pre-cloración & \\
\hline Fallas en el sistema de cloración & $\begin{array}{l}\text { Mantenimiento preventivo de equipos. } \\
\text { Disponer de equipos alternos. }\end{array}$ & $\begin{array}{l}\text { Utilizar los sistemas alternos: bombas auxiliares (02) } \\
\text { Utilizar sistema de dosificación por emergencia con } \\
\text { cilindros de } 907 \mathrm{~kg} \text {. }\end{array}$ \\
\hline \multirow[t]{2}{*}{ Falta de corriente eléctrica } & Mantenimiento de grupo electrógeno. & Hacer uso de grupo electrógeno. \\
\hline & Subproceso: Coagulación & \\
\hline Fallas en las bombas dosificadoras & Mantenimiento preventivo de equipos. & Instalación de bomba de contingencia. \\
\hline \multirow[t]{2}{*}{ Falta de disponibilidad de insumos } & Realizar compras anticipadas de insumos. & $\begin{array}{l}\text { Contar con coagulantes alternativos: cloruro férrico, } \\
\text { sulfato de aluminio solución y granular, sulfato férrico. }\end{array}$ \\
\hline & Subproceso: Post cloración & \\
\hline Fallas en el sistema de cloración & $\begin{array}{l}\text { Mantenimiento preventivo de equipos. } \\
\text { Disponer de equipos alternos. }\end{array}$ & $\begin{array}{l}\text { Utilizar los sistemas alternos: bombas auxiliares (02). } \\
\text { Utilizar con sistema de dosificación por emergencia } \\
\text { con cilindros de } 907 \mathrm{~kg} \text {. }\end{array}$ \\
\hline Falta de corriente eléctrica & Mantenimiento preventivo de grupo electrógeno. & Hacer uso de grupo electrógeno. \\
\hline
\end{tabular}

Fuente: Elaboración Propia.

Esta investigación pueda servir de base para la elaboración de los planes de continuidad de negocio en las empresas de saneamiento de agua, ya sea que están en la obligación de elaborar o no de elaborar dichos documentos.

La principal limitación para esta investigación ha sido que la documentación consultada sobre continuidad operativa se enfoca en el ámbito de la tecnología de la información.

\section{Agradecimientos}

A las Srtas. Karen Castillo y Jhois Maza, quienes contribuyeron al levantamiento de la información de campo de la empresa de estudio para la elaboración del plan de continuidad operativa.

Fuente de Financiamiento:

Consorcio Ópalo Andino E.I.R.L.(COPAN) 


\section{REFERENCIAS}

1. Belaunde G. Continuidad del Negocio u Operativa: Importancia del Análisis Estructural de Edificios. Gestión [en línea]. 11-01-2018 [citado ago 2018]. Disponible en https://gestion.pe/blog/riesgosfinancieros/2018/01/continuidad-del-negocio-u-operativa-importancia-del-analisis-estructural-deedificios.html

2. Atalait. Plan de Continuidad de Operaciones: todo lo que debes saber [en línea]. México, DF: Atalait; 2017 [citado jul 2018]. Disponible en: https:// www.atalait.com/blog/plan-de-continuidad-de-operaciones

3. Servicio Nacional de Aprendizaje-SENA. Curso: Planes de Emergencia; Unidad 1: Generalidades y Aspectos Legales. Bogotá: SENA; 2013.

4. De la Torre A. Plan de continuidad y plan de contingencia, una forma de salvar tu negocio. En: Planes de contingencia y planes de Continuidad del negocio. SCProgress [en línea]. 2017 [citado sep 2018]; [jul]:3-4. Disponible en: http://www.scprogress.com/NOTICIAS/CyberNoticia46-20170727.pdf

5. Redlnfo. Diseño e implementación de planes de continuidad de negocio [en línea]. Santiago de Chile: Redlnfo; [sd] [citado oct 2018]. Disponible en: http://www.redinfo.cl/bcp/

6. Borghello C. Plan de Continuidad Vs. Plan de Contingencias. Segulnfo [blog]. 17 de ago 2010 [citado sep 2018]. Disponible en: https://blog.segu-info. com.ar/2010/08/plan-de-continuidad-vs-plan-de.html

7. Alexander A. Nuevo Estándar Internacional en Continuidad del Negocio; ISO 22301:2012. Gestión [en línea] 2012 [citado jun 2018]; (oct-dic):00-06. [Disponible en: https://www.gestion.com.do/pdf/018/018-nuevo-estandarinternacional.pdf

8. UrbiCAD Architecture. Plan de Continuidad de Operaciones [en línea]. Valencia: Urbicad; [sd] [citado oct 2018]. Disponible en: http://www.safetymanagement.eu/PDF/normas_bcm.pdf

9. Sáez V. Modelo Integral para la implementación de un Plan de Continuidad de Negocio en Chile [tesis] [en línea]. Puerto Montt: Universidad Austral de Chile; 2013 [citado oct 2018]. Disponible en: http://cybertesis.uach.cl/tesis/ uach/2013/bpmfcis127m/doc/bpmfcis127m.pdf

10. Foro de Profesionales Latinoamericanos de Seguridad. Planes de contingencia. Qué es un plan de contingencia y cuáles son sus funciones, objetivos y contenidos [en línea]. [Buenos Aires]: Fuente original sitio Web de Belt Ibérica, www.belt.es; 2018 [citado oct 2018]. Disponible en: http://www. forodeseguridad.com/artic/segcorp/7209-que-es-un-plan-de-contingencia. htm

11. Positiva Compañia de Seguros ARL. Plan de Emergencia y Contingencias del Instituto Distrital de Deporte de Bogotá D.C [en línea]. Bogotá: Positiva Compañía de Seguros; 2015 [citado oct 2018]. Disponible en: http://www. idrd.gov.co/sitio/idrd/sites/default/files/imagenes/CBN-1107_0.pdf

12. Ferrer R. Plan de Continuidad de Negocio; Metodología recomendada para pequeños negocios [borrador] [en línea]. [Bogotá: SISTESEG]; 2010 [citado sep 2018]. Disponible en: http://www.sisteseg.com/files/ DocumentometodologiaBCPyDRP.pdf

13. OPS-OMS. Plan de Continuidad de Operaciones PWR Perú [en línea]. [Washington]: OPS-OMS; 2015 [citado oct 2018]. Disponible en: http:// bvsper.paho.org/videosdigitales/BCP-PER-2015.pdf
14. Instituto Nacional de Ciberseguridad-INCIBE. Plan de Contingencia y Continuidad del Negocio [en línea]. Madrid: INCIBE; [sd] [citado oct 2018]. Disponible en: https://www.incibe.es/sites/default/files/contenidos/ dosieres/metad_plan_de_contingencia_y_continuidad_de_negocio.pdf

15. Dirección de Prevención y Atención de Emergencias-DPAE. Guía para Elaborar planes de Emergencia y Contingencia [en línea]. Bogotá: DPAECámara de Comercio de Bogotá; 2009 [citado sep 2018. Disponible en: http://bibliotecadigital.ccb.org.co/bitstream/handle/11520/14249/ Gu\%C3\%ADa\%20para\%20elaborar\%20planes\%20de\%20emergencia. pdf?sequence $=1$ CtisAllowed $=y$

\section{Referencias complementarias:}

\section{Legislación peruana:}

Perú. Gobierno Revolucionario. Decreto Ley N 19338-Ley del Sistema Nacional de Defensa Civil-SINADECI. Lima: Gobierno de la República; 1972.

Perú. Ministerio de Economía y Finanzas. Ley № 29664: Ley del Sistema Nacional de Gestión de Riesgo de Desastre. Lima: Secretaria de Gestión del Riesgo de Desastres-Presidencia del Consejo de Ministros; 2011.

Perú. Presidencia del Consejo de Ministros. Lineamientos para la Gestión de Continuidad Operativa de la Entidades Públicas en los Tres Niveles de Gobierno, aprobado mediante la Resolución Ministerial № 028-2015-PCM. Lima: PCM; 2015.

Perú. Presidencia de la República. Reglamento de la Ley Nº 29664: Ley del Sistema Nacional de Gestión de Riesgo de Desastre-SINAGERD, aprobado mediante Decreto Supremo Nº 048-2011-PCM. Lima: Ministerio de Justicia; 2011.

Perú. Presidencia de la República. Reglamento del Sistema Nacional de Defensa Civil, aprobado mediante Decreto Supremo $N^{\circ}$ 005-88-SGMD. Lima: Presidencia de la República; 1988.

Normas:

BS 25999-1. Gestión de Continuidad del Negocio. Código de práctica. Calidad. 2006.

BS 25999-2 Gestión de Continuidad del Negocio. Especificación. 2007.

BS 25777 Gestión de Continuidad de la Tecnología de la Información y Comunicaciones. Código de prácticas. 2008.

ISO 22301 Seguridad de la Sociedad: Sistemas de Continuidad del Negocio. Requisitos. 2012.

ISO/IEC 27001 Sistemas de gestión de seguridad de la información. Requisitos. 2013.

ISO/IEC 27002 Sistemas de gestión de seguridad de la información. Código de práctica. 2013.

ISO/PAS 22399 Lineamientos sobre preparación para incidentes y gestión de continuidad operativa. 2007.

NFPA 1600 Norma sobre el Manejo de Desastres, Emergencias y Programas para la Continuidad de los Negocios. 2007. 